\title{
Screening for Genetic Variants Associated with Cardiovascular Diseases in Patients with Type 2 Diabetes Mellitus
}

\author{
Mihaleva Ivelina \\ Medical University Sofia \\ Mincheva Maria \\ Medical University Sofia \\ Hristova-Savova Mariela \\ Medical University Sofia

\section{Petkova Veronika} \\ Medical University Sofia

\section{Nikolov Rumen} \\ Medical University Sofia
}

\section{Tzvetanka Markova}

Medical University Sofia

\section{Gateva Pavlina}

Medical University Sofia

Ivanka Dimova ( $\square$ ivanka.i.dimova@gmail.com )

Medical University of Sofia Faculty of Medicine

\section{Research article}

Keywords: diabetes mellitus, cardiovascular disease, $\beta$-fibrinogen genotype -455G/A

Posted Date: December 28th, 2020

DOI: https://doi.org/10.21203/rs.3.rs-51215/v2

License: (1) (1) This work is licensed under a Creative Commons Attribution 4.0 International License. Read Full License 


\section{Abstract \\ Background}

Diabetes mellitus is associated with a wide range of cardiovascular diseases that comprise the largest cause of both morbidity and mortality for the diabetic patients. Our objective was to study the allelic and genotypic frequencies of genetic variants that have shown a strong association with cardiovascular disease in diabetic patients with and without cardiovascular complications and to assess the additional contribution of genetic variation in determining the risk for such complications.

\section{Methods}

We have used cardiovascular disease StripAssay kit (Vienna Lab) based on polymerase chain reaction and reverse hybridization. The following mutations were studied: FV G1691A (Leiden), FV H1299R (R2), Prothrombin G20210A, Factor XIII V34L, $\beta$-Fibrinogen - 455 G-A, PAI-1 4G/5G, GPIIla L33P (HPA-1), MTHFR C677T, MTHFR A1298C, ACE I/D, Apo B R3500Q, Apo E2/E3/E4. 36 diabetic patients divided in 2 groups were analyzed: 1) 20 diabetic patients with cardiovascular disease and 2) 16 diabetic patients without cardiovascular disease.

\section{Results}

We found higher than population frequency for the following alleles/genotypes - 5.5\% for FV Leiden allele, $9.7 \%$ for FVR2 allele, 38.9\% for $\beta$-Fibrinogen genotype $-455 \mathrm{G} / \mathrm{A}, 58.9 \%$ for PAI- $14 \mathrm{G}$ allele, $36.1 \%$ for ACE D/D genotype. Statistically higher frequency was established for $\beta$-Fibrinogen - $455 \mathrm{G}-\mathrm{A}$ in the patients with cardiovascular disease compared to non- cardiovascular disease (55\% vs. $18.7 \%$ ).

\section{Conclusions}

We detected high frequency of $\beta$-Fibrinogen - $455 \mathrm{G} / \mathrm{A}$ genotype in diabetic patients, especially in these with cardiovascular disease. Based on its pro-inflammatory role and its connection to possible thrombotic events, patients would benefit from anti-inflammatory treatment.

\section{Introduction}

Diabetes mellitus (DM) is a very serious health issue that has reached extremely high levels worldwide nowadays. Almost half a billion people are living with the disease. Findings of the current $9^{\text {th }}$ edition of the International Diabetes Federation (IDF) atlas state that DM is one of the diseases that grows very fast all around the world. It is found that in 2019463 million people have diabetes and this number is expected to reach 578 million by 2030, and 700 million by 2045. Unfortunately, three out of four patients are of working age and over 4 million people of the age 20-79 years are about to die from diabetes- 
related causes in 2019. There is a 15\% increase in the people with diabetes in Europe - they are 59 million in 2019 and their number is expected to rise to 66 million in 2030 and to 68 million in 2040, respectively. Type 2 DM accounts for the vast majority - around $90 \%$ - of the disease worldwide (1).

It is well known that the long-term complications of diabetes can be present at diagnosis in people with type 2 diabetes. The disease is associated with a wide range of cardiovascular disease (CVD) that comprise the largest cause of both morbidity and mortality for the patients (2). The prevalence of coronary artery disease (CAD) is found to be around $21 \%$ and that of any CVD is around $32 \%$ in adults with diabetes (3). The morbidity from CVD in diabetic patients is 2 to 4 fold higher in comparison to people without diabetes. Patients with DM without myocardial infarction (MI) have exactly the same risk for CAD as people that have already have MI (4). The most common types of CVD that are found in patients with diabetes are arterial hypertension, coronary heart disease, cerebrovascular disease, peripheral artery disease as well as congestive heart failure. As a whole, CVD contribute for between onethird and one-half of all deaths.

Metabolic syndrome is a specific set of symptoms, which plays an important role in cardiovascular morbidity and mortality. It is a progressive phenotype which is characterized by insulin resistance, abdominal obesity, hypertension, dyslipidemia or type $2 \mathrm{DM}$. As it is known, atherosclerosis is a chronic inflammatory and lipid-depository disease that can eventually lead to different cardiovascular events. Subclinical inflammation is observed in type $2 \mathrm{DM}$, obesity, insulin resistance. It is characterized by overexpression of cytokines which are produced by adipocytes, activated macrophages and other cells. Inflammatory mediators like plasminogen activator inhibitor - 1 (PAl-1), C-reactive protein (CRP), fibrinogen and others take part in signal pathways, in insulin action and in amplifying the inflammatory response. These cytokines are connected also with chronic inflammatory processes which cause lipid accumulation and development of atherosclerosis and CVD. Atherosclerosis is a complex multifactorial disease and the accelerating of the atherosclerotic process in DM may be explained by hyperglycemia, oxidative stress, accumulation of advanced glycation end products (AGEs), dyslipidemia, hyperinsulinemia, overexpression of inflammatory markers and genetic variabilities (5).

Methylenetetrahydrofolate reductase (MTHFR) catalyzes the conversion of 5,10methylenetetrahydrofolate in 5-methyltetrahydrofolate. MTHFR plays role in the metabolism of folate and in the homeostasis of homocysteine. Frequent C677T polymorphism in MTHFR is connected with high risk of development of CVD, DM. The mutation leads to hyperhomocysteinemia - a risk factor for atherosclerosis (6). On the other hand, it is found that a number of common polymorphisms and mutations in the genes coding for Factor V Leiden (FVL) and MTHFR can contribute to deep vein thrombosis - a condition that can be associated with hypercoagulability, which can be genetic or acquired. A study determines the prevalence of FVL, MTHFR C677T and MTHFR A1298C gene polymorphisms in patients with the disease. The results show that MTHFR A1298C gene was found in 77\% among cases, followed by MTHFR C677T (67\%) and FVL (17\%) (7). 
Renin-angiotensin-aldosterone system (RAAS) regulates the blood volume and pressure. It also has a role in the pathogenesis of atherosclerosis and can take part in the development of arterial hypertension, insulin resistance, DM, obesity, vascular and systemic inflammation. Angiotensin II activates intracellular signal pathways which cause atherothrombosis through inflammation, endothelial dysfunction, impaired fibrinolysis. Genetic polymorphism of RAAS genes including of angiotensin converting enzyme (ACE), angiotensin II type I receptor, angiotensinogen take part in atherosclerosis pathogenesis (8). The DD genotype of ACE is known to be connected to higher serum activity of ACE as well as the risk of left ventricular hypertrophy, arterial hypertonia and $\operatorname{CAD}$ occurrence $(9,10)$.

Plasma lipoproteins are made of hydrophobic core that consists of triglycerides and cholesterol esters, and of superficial monolayer of phospholipids, unesterified cholesterol and apolipoprotens. Increased levels of apolipoprotein B (apoB) - containing lipoproteins like LDL and chylomicron remnants cause atherosclerosis. Chylomicrons which contain apoB 48 are secreted in guts after meal while VLDL which have apoB 100 come from the liver. The metabolism of chylomicron remnants and VLDL in liver is facilitated by apolipoprotein $E$ (apoE). ApoB 100 is responsible for LDL uptake in liver (11). Genetic defect of apoB 100 causes increased level of LDL which accumulates in plasma and leads to hypercholesterolemia and premature atherosclerosis. On the other hand, patients which lack apoE accumulate lipoprotein remnants. Lipoprotein remnants with apoE stimulate accumulation of cholesterol esters in macrophages. In lesions most of apoE molecules are synthesized locally by macrophages (12).

Different studies evaluate hemostatic gene variants and atherothrombotic and cardiovascular complications. Diabetic patients are affected by abnormalities of the coagulation cascade and are predisposed to thrombotic events because of metabolic changes and acquired or inherited coagulation defects (13). Factor V (FV) Leiden is a procoagulant mutation that is associated with venous and arterial thrombosis as well as pregnancy complications. The relationship between the factor $\mathrm{V}$ Leiden mutation and atherosclerosis is a matter of debate due to conflicting data. A study found a relevant increase in the prevalence of diabetes between patients with venous thromboembolism carriers of FVL compared to noncarriers of FVL although this was not statistically significant (14). Persistent hyperglycaemia in diabetes mellitus causes coagulopathies due to glycation of haemoglobin, prothrombin, fibrinogen and other proteins that are involved in the clotting pathway. Shortened activated partial thromboplastin time (aPTT) and prothrombin time (PT) reflect hypercoagulable state, which is associated with an increased thrombotic risk and different CVD (15). Plasminogen activator inhibitor-1 (PAl-1) also known as endothelial plasminogen activator inhibitor or serpin E1 is a serine protease inhibitor (serpin) that functions as the main inhibitor of tissue plasminogen activator (tPA) and urokinase (UPA), the activators of plasminogen and the process of fibrinolysis. Elevated PAI- 1 is an important risk factor for thrombosis and atherosclerosis (16). Circulating PAI-1 levels are found to be elevated in patients with CAD. There are studies that show that insulin resistance may be a regulator of PAl-1 expression. The production of PAl-1 by adipose could be an important contributor to the elevated plasma PAl-1 levels that are seen in patients with insulin resistance (17). Patients with metabolic syndrome typically present with significantly higher levels of PAI-1 (18). Prospective studies of patients with MI or CAD have showed the association between increased plasma PAl-1 levels and the risk of coronary events (17). A recent metaanalysis has also 
proved that PAl-1 polymorphism (4G/5G) is associated with $\mathrm{MI}(19)$. PAl -1 is linked to RAAS too, which is an important contributor to vascular disease initiation and progression (20). Small drug molecules have been developed for PAl-inhibition. Tiplaxtinin, (PAI-039), and piperazine-chemotype molecules have been studied (21). Small molecules anti-PAI-1 that are orally bioavailable as TM5001, TM5007, TM5275, were tested in animal models, with some in vitro good results, but unfortunately they did not achieve enough data to be used (22). Factor XIII or fibrin stabilizing factor that is activated by thrombin to factor XIIIa. XIIla is an enzyme of the blood coagulation system that crosslinks fibrin. Deficiency of XIII increases bleeding tendency (23). Human platelet antigens (HPA) are polymorphisms in platelet antigens. Platelets play a very important role in acute arterial occlusion and platelet hyperactivity may contribute to an increased risk for CVD. Platelets attach to subendothelial structures by specific receptors such as the collagen receptor glycoprotein (GP) la/lla, or the primary von Willebrand factor (VWF) receptor GPIb/IX. After that they become activated and aggregate by cross-linking via the fibrinogen receptor GPIllb/llla (24). There is data that GPIIla (HPA-1) may play a protective role in CVD. Iniesta et al. found that the platelet GP IIla Polymorphism HPA-1 protects against subarachnoid hemorrhage and that the suggested platelet hyper-reactivity that is determined by this allele could reduce the risk to suffer that disease (25).

Fibrinogen (factor I) is a glycoprotein complex that is produced by the liver. In case of tissue and vascular injury it is converted by thrombin to fibrin and then to a fibrin-based blood clot which acts to occlude blood vessels and stop bleeding. Fibrinogen is a "positive" acute-phase protein and its blood levels rise in response to certain conditions like systemic inflammation or tissue injury (26). Studies have shown that high levels of fibrinogen are associated with $C A D$ and may contribute to vascular disease by increasing blood viscosity thus stimulating fibrin formation, or increasing platelet-platelet interaction (27). Fibrinogen is considered as being involved in thrombotic occlusion and in the final stage of atherothrombosis. There are studies suggesting that fibrinogen may play a more active role in the development and progression of atherosclerotic plaque (28). On the other hand, fibrinogen production and plasma concentration are increased in type $2 \mathrm{DM}$. It is not known whether altered response to insulin contributes to hyperfibrinogenemia in diabetic patients. Fibrinogen production is acutely increased by insulin when euglycemia and euaminoacidemia are maintained in type 2 diabetic individuals but not in people without the disease. Enhanced fibrinogen production by insulin is supposed to be a main alteration leading to hyperfibrinogenemia and to cardiovascular risk in type 2 DM (29). Fibrinogen expression and deposition is also increased in obese people. The increase in fibrinogen expression and fibrin deposition leads to increased adipocyte inflammation and macrophage infiltration which suppresses glucose uptake and may lead to adipose tissue fibrosis. However, relationship between fibrinogen and insulin resistance is controversial. Free fatty acids may explain the relationship between fibrinogen and insulin resistance because a simultaneous increase in free fatty acids and fibrinogen is seen in variety of clinical and experimental condition. This relationship might also result from an inflammatory reaction that accompanies atherosclerosis (30). A common mutation $-455 \mathrm{G} / \mathrm{A}$ in the promoter region of the beta-fibrinogen gene has been associated with elevated fibrinogen in plasma. Carter et al. studied the association of G/A polymorphism at position -455 of the beta-fibrinogen gene and fibrinogen levels in the development of CAD in people with non-insulin dependent DM. The results 
showed that fibrinogen levels were significantly higher in the patients with CAD than those without. The data suggested a relationship between the $-455 \mathrm{G} / \mathrm{A}$ beta-fibrinogen gene polymorphism and the development of CAD in DM (31). Lam et al. investigated the relation between the G/A-b-fibrinogen gene polymorphism and plasma fibrinogen concentration and its role in CAD in patients with type $2 \mathrm{DM}$ and in non-diabetic control subjects. They concluded that the G/A455 polymorphism of the b-fibrinogen gene is a genetic determinant of plasma fibrinogen concentrations and CAD in their cohort (32).

\section{Aim of the study}

To investigate the allelic and genotypic frequencies of genetic variants that have shown strong association with CVD in patients with type 2 DM and the presence or absence of cardiovascular complications in order to estimate the additional contribution of the genetic variations in determining the risk of such complications.

\section{Materials And Methods}

We collected probes from peripheral venous blood of patients with type 2 diabetes mellitus. They were divided in two groups according to their cardiovascular status: i) with type 2 DM and CVD, middle age $56,3 \pm 10,8$, and ii) with type 2 DM without CVD, middle age $42,5 \pm 10,8$. Their general characteristics are shown on table 1.

\begin{tabular}{|lll|}
\hline Parameters & Type 2 DM withCVD & Type 2 DM without CVD \\
\hline Age (years) & $56,3 \pm 10,8$ & $42,5 \pm 10,8$ \\
\hline BMI $\left(\mathrm{kg} / \mathrm{m}^{2}\right)$ & $34.8 \pm 8.4$ & $38.1 \pm 13.7$ \\
\hline HbA1c $(\%)$ & $8.8 \pm 1.8$ & $7.9 \pm 2.3$ \\
\hline MetS (\%) & 77.8 & 66.7 \\
\hline
\end{tabular}

Table 1 - General characteristics of participants in the subgroups

The statistical analysis of the data was performed through SPSS v.20.0 (SPSS, Chicago, USA). The data are expressed as middle value \pm standard deviation (SD). Student's T-test and one-way analysis of variance (One-way ANOVA) are used for comparison of continuous variables and Pearson's chi-squared test for proportional comparisons. P-value less than 0,05 is statistically significant.

Our data do not show statistically significant difference in sex, middle age, BMI, $\mathrm{HbA1c}$, the presence of metabolic syndrome (MeS) so these variables cannot influence the results from the DNA analysis.

We have used CVD StripAssay kit (Vienna Lab) based on polymerase chain reaction (PCR) and reverse hybridization. The procedure included three steps: 1. DNA isolation; 2. PCA amplification with biotinized 
primers; 3 . Hybridization of amplificated products on test strip containing specific for the allele oligonucleotide probe immobilized on a massive of parallel bands (figure 1). The bound biotinized sequence are found with the help of streptavidin - alkaline phosphatase and colour substrates.

The following mutations were studied: FV G1691A (Leiden), FV H1299R (R2), Prothrombin G20210A, Factor XIII V34L, $\beta$-Fibrinogen 455 G/A, PAI-1 4G/5G, GPIIla L33P (HPA-1), MTHFR C677T, MTHFR A1298C, ACE D/D, Apo B R3500Q, Apo E2/E3/E4.

Principle of the test:

In vitro amplification (PCR; 2 separate reactions of a probe)

The amplificated products are stored in ice or at $2-8^{\circ} \mathrm{C}$ for later use.

Test with electrophoresis

Analysis of the products of amplification with gel electrophoresis (2,8\% agar gel).

Hybridization ( $45^{\circ} \mathrm{C}$; water bath system)

Result interpretation

The genotype of the probe is determined with the help of Collector TM sheet.

The processed Test strip is put in one of the fields, it is leveled up to the schematic draught with the help of the red marker line (top) and the green one (bottom) and is fixed with glue.

A positive reaction of the top control line shows the right function of Conjugate Solution and Color Developer. That line must always be positively coloured.

For each polymorphic position one of the following colour models must be present and the intensity of the positive lines may vary (figure 2).

For the three Apo E isoforms E2, E3 and E4 the following colour models can be seen:

The six possible homozygotic and heterozygotic Apo E genotypes (E2/2, E3/3, E4/4, E2/3, E2/4, E3/4) correspond to the shown bands.

The allelic frequencies of each of the investigated genetic variants were determined and were compared to the population frequencies from genomic databases - The Genome Aggregation Database (gnomAD), 1000 Genomes Project phase 3 database, Ensembl Genome Browser.

In our cohort the number of patients studied is 36 , that are 72 alleles - these are 20 patients from the first group ( 40 alleles) and 16 from the second one (32 alleles). For some genetic variants the number is less due to unsuccessful analysis. 


\section{Results}

Figure 4 and 5 show the results from the genotyping of 12 genetic variants in risk genes in diabetic patients with and without CVD.

\subsection{Results from the genotyping of Factor V Leiden and HR2}

Altogether for all patients a frequency of $5,5 \%$ is found - more than two-fold increase than the world population frequency of $1,9 \%$ and $2,9 \%$ in Europe. According to 1000 Genomes database the frequency of the heterozygotes is $2 \%$ and in our cohort we found it $11 \%$. No connection between the mutation of FV Leiden and cardiovascular complications has been established, even in the group of the patients without CVD a higher frequency of the mutation is found $-9,4 \%$. We found also a higher than population frequency for FV H1299R (R2) - 9,7\% in comparison to world frequency 5,7\% and $6 \%$ in Europe.

\begin{tabular}{|llll|}
\hline Allele/Genotype & DM with CVD & DM without CVD & All \\
\hline FV (Leiden) & $2.5 \%$ & $9.4 \%$ & $5.5 \%$ \\
\hline FV G/A & $5 \%$ & $18.8 \%$ & $11 \%$ \\
\hline FV A/A & 0 & 0 & 0 \\
\hline FV (R2) & $8.8 \%$ & $10.7 \%$ & $9.7 \%$ \\
\hline FV H/R & $17.6 \%$ & $21.4 \%$ & $19.4 \%$ \\
\hline FV R/R & 0 & 0 & 0 \\
\hline
\end{tabular}

Table 2 - Allelic and genotypic frequencies of FV G1691A (Leiden) and FV H1299R (R2)

\subsection{Results from the genotyping of Prothrombin}

We found a frequency of $1,4 \%$ and it is comparable to the world population frequency of $0,8 \%$ and to that in Europe $-1,1 \%$.

\begin{tabular}{|llll|}
\hline Allele/Genotype & DM with CVD & DM without CVD & All \\
\hline Prothrombin 20210A & $2.5 \%$ & 0 & $1.4 \%$ \\
\hline Prothrombin G/A & $5 \%$ & 0 & $2.8 \%$ \\
\hline Prothrombin A/A & 0 & 0 & 0 \\
\hline
\end{tabular}

Table 3 - Allelic and genotypic frequencies of Prothrombin G20210A 


\subsection{Results from the genotyping of PAI - 1}

We found higher frequency of the pathogenic allele - $58,6 \%$ compared to $26,9 \%$ world population frequency according to Ensambl and 38,9\% in Europe. The frequency of the homozygotes is $31,4 \%$ in comparison to $20,9 \%$ in the world and $29,4 \%$ in Europe.

\begin{tabular}{|llll|}
\hline Allele/Genotype & DM with CVD & DM without CVD & All \\
\hline PAl-1 4G & $65 \%$ & $50 \%$ & $58.6 \%$ \\
\hline PAl-1 4G/5G & $60 \%$ & $46.7 \%$ & $54.3 \%$ \\
\hline PAl-1 4G/4G & $35 \%$ & $26.7 \%$ & $31.4 \%$ \\
\hline
\end{tabular}

Table 4 - Allelic and genotypic frequencies of PAI-1 4G/5G

\subsection{Results from the genotyping of Factor XIII}

In our cohort was found lower frequency of the minor allele of $11,1 \%$ compared to $21,9 \%$ world population frequency and $25,2 \%$ in Europe. It is important to note that in the group with CVD the frequency is even lower $-7,5 \%$, which suggests a protective role of that genetic variant.

\begin{tabular}{|llll|}
\hline Allele/Genotype & DM with CVD & DM without CVD & All \\
\hline Factor XIII 34L & $7.5 \%$ & $15.6 \%$ & $11.1 \%$ \\
\hline Factor XIII V/L & $15 \%$ & $31.2 \%$ & $22.2 \%$ \\
\hline Factor XIII L/L & 0 & 0 & 0 \\
\hline
\end{tabular}

Table 5 - Allelic and genotypic frequencies of Factor XIII V34L

\subsection{Results from the genotyping of $\underline{\beta-F i b r i n o g e n}$}

The allelic frequency in our group is $22,2 \%$ which is higher than the world population frequency $-16,9 \%$, and that in Europe $-20,3 \%$. According to 1000 Genomes database the population frequency of the heterozygotes is $22 \%$ and we found it $38,9 \%$. It increases statistically significant in the group with CVD compared to the one without CVD - 55\% versus $18,7 \%$ - figure 11 . 


\begin{tabular}{|llll|}
\hline Allele/Genotype & DM with CVD & DM without CVD & All \\
\hline$\beta$-Fibrinogen - 455 A & $27.5 \%$ & $15.6 \%$ & $22.2 \%$ \\
\hline$\beta$-Fibrinogen - 455 G/A & $\begin{array}{l}\mathbf{5 5 \%} \\
\mathbf{p}<0.03\end{array}$ & $18.7 \%$ & $38.9 \%$ \\
\hline$\beta$-Fibrinogen -455A/A & 0 & $6.2 \%$ & \\
\hline
\end{tabular}

Table 6 - Allelic and genotypic frequencies of $\beta$-Fibrinogen - $455 \mathrm{G} / \mathrm{A}$

\subsection{Results from the genotyping of HPA1}

The found from us allelic frequency is $12,5 \%$ and is comparable to the world population frequency $12,1 \%$; in Europe - 15,2\%.

\begin{tabular}{|llll|}
\hline Allele/Genotype & DM with CVD & DM without CVD & All \\
\hline HPA-1b & $7.5 \%$ & $18.7 \%$ & $12.5 \%$ \\
\hline HPA-1a/1b & $15 \%$ & $37.4 \%$ & $25 \%$ \\
\hline HPA-1b/1b & 0 & 0 & 0 \\
\hline
\end{tabular}

Table 7 - Allelic and genotypic frequencies of GPIIla L33P (HPA-1)

In order to conclude about the factors contributing to congenital thrombophilia we found higher frequencies for most of them than in the world population frequency but not reaching statistical significance. The highest frequency is that of the PAl-1 variant in patients with DM. The frequency of Factor XIII polymorphism is lower than that in world population frequency which is in accordance to the suggested protective role of the polymorphism. When comparing the frequencies in the groups with and without CVD only the variants of PAI-1 and Fibrinogen show higher frequency in the group with CVD figure 13.

\subsection{Results from the genotyping of MTHFR}

The allelic frequency of MTHFR 677T we found is $25 \%$ and is a little lower than that of world population $-31 \%$, and in Europe $-32 \%$. The allelic frequency of MTHFR $1298 \mathrm{C}$ in our study is $38,9 \%$ and is higher than that in world $-29 \%$, and in Europe $-32 \%$. 


\begin{tabular}{|llll|}
\hline Allele/Genotype & DM with CVD & DM without CVD & All \\
\hline MTHFR 677T & $22.5 \%$ & $28.1 \%$ & $25 \%$ \\
\hline MTHFR C/T & $25 \%$ & $31.2 \%$ & $27.8 \%$ \\
\hline MTHFR T/T & $10 \%$ & $12.5 \%$ & $11.1 \%$ \\
\hline MTHFR 1298C & $47.5 \%$ & $28.1 \%$ & $38.9 \%$ \\
\hline MTHFR A/C & $55 \%$ & $31.2 \%$ & $44.4 \%$ \\
\hline MTHFR C/C & $20 \%$ & $12.5 \%$ & $16.7 \%$ \\
\hline
\end{tabular}

Table 8 - Allelic and genotypic frequencies of MTHFR C677T и MTHFR A1298C

\subsection{Results from the genotyping of ACE}

We found a frequency of the homozygotes of the pathologic allele that is $36,1 \%$ and is higher than population frequency in Europe - 25\%.

\begin{tabular}{|llll|}
\hline Allele/Genotype & DM with CVD & DM without CVD & All \\
\hline ACE Del & $55 \%$ & $65.6 \%$ & $59.7 \%$ \\
\hline ACE I/D & $50 \%$ & $43.7 \%$ & $47.2 \%$ \\
\hline ACE D/D & $30 \%$ & $43.7 \%$ & $36.1 \%$ \\
\hline
\end{tabular}

Table 9 - Allelic and genotypic frequencies of ACE I/D

\subsection{Results from the genotyping of $A p o B$}

The mutation was not found in any of the patients and its world population frequency is 1:5000.

1.10. Results from the genotyping of ApoE

The frequency of the risk allele E4 we found is $13,9 \%$ and is comparable to the world population frequency - 13,8\%q and that in Europe - 16,1\% 


\begin{tabular}{|llll|}
\hline Allele/Genotype & DM with CVD & DM without CVD & All \\
\hline Apo E3/E4 & $10 \%$ & $18.7 \%$ & $13.9 \%$ \\
\hline Apo E2/E4 & $5 \%$ & 0 & $2.8 \%$ \\
\hline Apo E3/E3 & $85 \%$ & $81.3 \%$ & $83.3 \%$ \\
\hline
\end{tabular}

Table 10 - Allelic and genotypic frequencies of Apo E2/E3/E4

\section{Discussion}

The investigation of the allelic frequency of 12 genetic variants connected to cardiovascular risk in Bulgarian patients with type 2 DM showed more than two-fold increase in comparison to population frequency for the following alleles:

- 5,5\% for FV (Leiden) mutation compared to 1,9\% in world population and 2,9\% in Europe population (no connection between FV (Leiden) mutation and cardiovascular complications has been established - in the group of patients without CVD the frequency of the mutation is higher $-9,4 \%$ compared to $2,5 \%$ in the group of patients with CVD)

- $58,6 \%$ for PAl- $14 \mathrm{G}$ in comparison to $26,9 \%$ in world population

Increased frequency in comparison to world population for the following genotypes has been found:

- $38,9 \%$ for $\beta$-fibrinogen $455 \mathrm{G} / \mathrm{A}$ compared to $22 \%$ in world population

- $36,1 \%$ for ACE D/D compared to $25 \%$ in world population

Lower frequency for Factor XIII 34L and MTHFR C677T in the investigated groups in comparison to world population has been found. It is suggested that Factor XIII 34L may have protective role in CVD development.

No statistically significant difference between the investigated groups with and without CVD in the allelic and genotypic frequency in 11 out of 12 studied genetic variants has been found.

A statistically significant higher frequency in heterozygotes for $\beta$-fibrinogen $455 \mathrm{G} / \mathrm{A}$ in the group of patients with DM and CVD has been found $-55 \%$ in comparison to $18,7 \%$ in the group without CVD. It is supposed that the role of $\beta$-fibrinogen as pro-inflammatory protein along with its thrombotic effects may increase the risk for CVD in patients with DM.

\section{Conclusion}

In our study we aimed at investigating the allelic and genotypic frequencies of genetic variants that have are supposed to have strong association with CVD in patients with type 2 DM with and without 
cardiovascular complications in order to try to estimate the additional contribution of the genetic variations in determining the risk of such complications. We found a statistically significant higher frequency in heterozygotes for $\beta$-fibrinogen $455 \mathrm{G} / \mathrm{A}$ in the group of patients with DM and CVD which is also seen in other studies. This comes to show that fibrinogen is really an important contributor to the pathogenesis of CVD, especially in patients with type $2 \mathrm{DM}$.

\section{Declarations}

\section{Ethics approval and consent to participate:}

The collection of patients' samples was approved by the institutional ethical committee (Medical University Sofia) with the approval No1209/2018. Each patient signed a written Informed consent.

\section{Consent for publication:}

It is included in the text of the Informed consent signed by the patient. All participants in the study signed the Informed consent.

\section{Availability of data and materials:}

All data and material are available in the Molecular Medicine Centre, Medical University Sofia

\section{Competing interests:}

No

\section{Funding:}

The study was supported by Grant of Bulgarian National Scientific Foundation with No KП-06-H 33/10, 2019

\section{Authors' contributions:}

All authors contributed to the study conception and design. Methodology: IM, MM, MH, VP, ID; Formal analysis and investigation: IM, PG, RN, TM, ID; Writing - original draft preparation: IM, ID; Writing - review and editing: PG, RN, TM; Funding acquisition: ID; Resources: ID; Supervision: ID

\section{Acknowledgements -}

The study was supported by Grant of BNSF with No KП-06-ПН 33/10, 2019 


\section{References}

1. Saeedi P, Petersohn I, Salpea P, Malanda B, Karuranga S, Unwin N, et al. Global and regional diabetes prevalence estimates for 2019 and projections for 2030 and 2045: Results from the International Diabetes Federation Diabetes Atlas, 9(th) edition. Diabetes research and clinical practice. 2019;157:107843.

2. Gerstein HC. Diabetes: Dysglycaemia as a cause of cardiovascular outcomes. Nature reviews Endocrinology. 2015;11(9):508-10.

3. Einarson TR, Acs A, Ludwig C, Panton UH. Prevalence of cardiovascular disease in type 2 diabetes: a systematic literature review of scientific evidence from across the world in 2007-2017. Cardiovascular diabetology. 2018;17(1):83.

4. Bulugahapitiya U, Siyambalapitiya S, Sithole J, Idris I. Is diabetes a coronary risk equivalent? Systematic review and meta-analysis. Diabetic medicine : a journal of the British Diabetic Association. 2009;26(2):142-8.

5. Ait-Oufella H, Taleb S, Mallat Z, Tedgui A. Recent advances on the role of cytokines in atherosclerosis. Arteriosclerosis, thrombosis, and vascular biology. 2011;31(5):969-79.

6. Liew SC, Gupta ED. Methylenetetrahydrofolate reductase (MTHFR) C677T polymorphism: epidemiology, metabolism and the associated diseases. European journal of medical genetics. 2015;58(1):1-10.

7. Ehsani M, Imani A, Moravveji A. Prevalence of factor V leiden, MTHFR C677T and MTHFR A1298C polymorphisms in patients with deep vein thrombosis in Central Iran. Molecular biology reports. 2018;45(4):621-4.

8. Durante A, Peretto G, Laricchia A, Ancona F, Spartera M, Mangieri A, et al. Role of the reninangiotensin-aldosterone system in the pathogenesis of atherosclerosis. Current pharmaceutical design. 2012;18(7):981-1004.

9. Hernandez D, de la Rosa A, Barragan A, Barrios Y, Salido E, Torres A, et al. The ACE/DD genotype is associated with the extent of exercise-induced left ventricular growth in endurance athletes. Journal of the American College of Cardiology. 2003;42(3):527-32.

10. Beohar N, Damaraju S, Prather A, Yu QT, Raizner A, Kleiman NS, et al. Angiotensin-I converting enzyme genotype DD is a risk factor for coronary artery disease. Journal of investigative medicine : the official publication of the American Federation for Clinical Research. 1995;43(3):275-80.

11. Morita SY. Metabolism and Modification of Apolipoprotein B-Containing Lipoproteins Involved in Dyslipidemia and Atherosclerosis. Biological \& pharmaceutical bulletin. 2016;39(1):1-24.

12. Fazio S, Babaev VR, Murray AB, Hasty AH, Carter KJ, Gleaves LA, et al. Increased atherosclerosis in mice reconstituted with apolipoprotein E null macrophages. Proceedings of the National Academy of Sciences of the United States of America. 1997;94(9):4647-52.

13. Dubsky M, Jirkovska A, Pagacova L, Bem R, Nemcova A, Fejfarova V, et al. Impact of Inherited Prothrombotic Disorders on the Long-Term Clinical Outcome of Percutaneous Transluminal 
Angioplasty in Patients with Diabetes. Journal of diabetes research. 2015;2015:369758.

14. Lodigiani C, Ferrazzi P, Di Micco P, Libre L, Genovese S, Quaglia I, et al. Is there a relationship between factor $V$ Leiden and type 2 diabetes? Journal of translational medicine. 2009;7:52.

15. Ephraim RK, Awuku YA, Adu P, Ampomah LT, Adoba P, Panford S, et al. High risk of coagulopathy among Type-2 Diabetes Mellitus clients at a municipal hospital in Ghana. Ghana medical journal. 2017:51(3):101-7.

16. Vaughan DE. PAl-1 and atherothrombosis. Journal of thrombosis and haemostasis : JTH. 2005;3(8):1879-83.

17. Juhan-Vague I, Alessi MC. PAI-1, obesity, insulin resistance and risk of cardiovascular events. Thrombosis and haemostasis. 1997;78(1):656-60.

18. Ito S, Iwaki S, Koike K, Yuda Y, Nagasaki A, Ohkawa R, et al. Increased plasma sphingosine-1phosphate in obese individuals and its capacity to increase the expression of plasminogen activator inhibitor-1 in adipocytes. Coronary artery disease. 2013;24(8):642-50.

19. Gong LL, Peng JH, Han FF, Zhu J, Fang LH, Wang YH, et al. Association of tissue plasminogen activator and plasminogen activator inhibitor polymorphism with myocardial infarction: a metaanalysis. Thrombosis research. 2012;130(3):e43-51.

20. Senchenkova EY, Russell J, Almeida-Paula LD, Harding JW, Granger DN. Angiotensin II-mediated microvascular thrombosis. Hypertension. 2010;56(6):1089-95.

21. Pandya V, Jain M, Chakrabarti G, Soni H, Parmar B, Chaugule B, et al. Discovery of inhibitors of plasminogen activator inhibitor-1: structure-activity study of 5-nitro-2-phenoxybenzoic acid derivatives. Bioorganic \& medicinal chemistry letters. 2011;21(19):5701-6.

22. Izuhara Y, Yamaoka N, Kodama H, Dan T, Takizawa S, Hirayama N, et al. A novel inhibitor of plasminogen activator inhibitor-1 provides antithrombotic benefits devoid of bleeding effect in nonhuman primates. Journal of cerebral blood flow and metabolism : official journal of the International Society of Cerebral Blood Flow and Metabolism. 2010;30(5):904-12.

23. Muszbek L, Bereczky Z, Bagoly Z, Komaromi I, Katona E. Factor XIII: a coagulation factor with multiple plasmatic and cellular functions. Physiological reviews. 2011;91(3):931-72.

24. Metcalfe P, Watkins NA, Ouwehand WH, Kaplan C, Newman P, Kekomaki R, et al. Nomenclature of human platelet antigens. Vox sanguinis. 2003;85(3):240-5.

25. Iniesta JA, Gonzalez-Conejero R, Piqueras C, Vicente V, Corral J. Platelet GP Illa polymorphism HPA-1 (PIA) protects against subarachnoid hemorrhage. Stroke. 2004;35(10):2282-6.

26. de Moerloose P, Casini A, Neerman-Arbez M. Congenital fibrinogen disorders: an update. Seminars in thrombosis and hemostasis. 2013;39(6):585-95.

27. Baser H, Can U, Baser S, Hidayetoglu BT, Aslan U, Buyuktorun I, et al. Serum total oxidant/antioxidant status, ischemia-modified albumin and oxidized-low density lipoprotein levels in patients with vitamin D deficiency. Archives of endocrinology and metabolism. 2015;59(4):318-24. 
28. Levenson J, Giral P, Razavian M, Gariepy J, Simon A. Fibrinogen and silent atherosclerosis in subjects with cardiovascular risk factors. Arteriosclerosis, thrombosis, and vascular biology. 1995;15(9):1263-8.

29. Barazzoni R, Kiwanuka E, Zanetti M, Cristini M, Vettore M, Tessari P. Insulin acutely increases fibrinogen production in individuals with type 2 diabetes but not in individuals without diabetes. Diabetes. 2003;52(7):1851-6.

30. Stegnar M. Disturbances of Haemostasis in Diabetes Mellitus Contributing to the Development of Atherosclerosis. Ejifcc. 2002;13(5):205-9.

31. Carter AM, Mansfield MW, Stickland MH, Grant PJ. Beta-fibrinogen gene-455 G/A polymorphism and fibrinogen levels. Risk factors for coronary artery disease in subjects with NIDDM. Diabetes care. 1996;19(11):1265-8.

32. Lam KS, Ma OC, Wat NM, Chan LC, Janus ED. Beta-fibrinogen gene G/A-455 polymorphism in relation to fibrinogen concentrations and ischaemic heart disease in Chinese patients with type II diabetes. Diabetologia. 1999;42(10):1250-3.

\section{Figures}




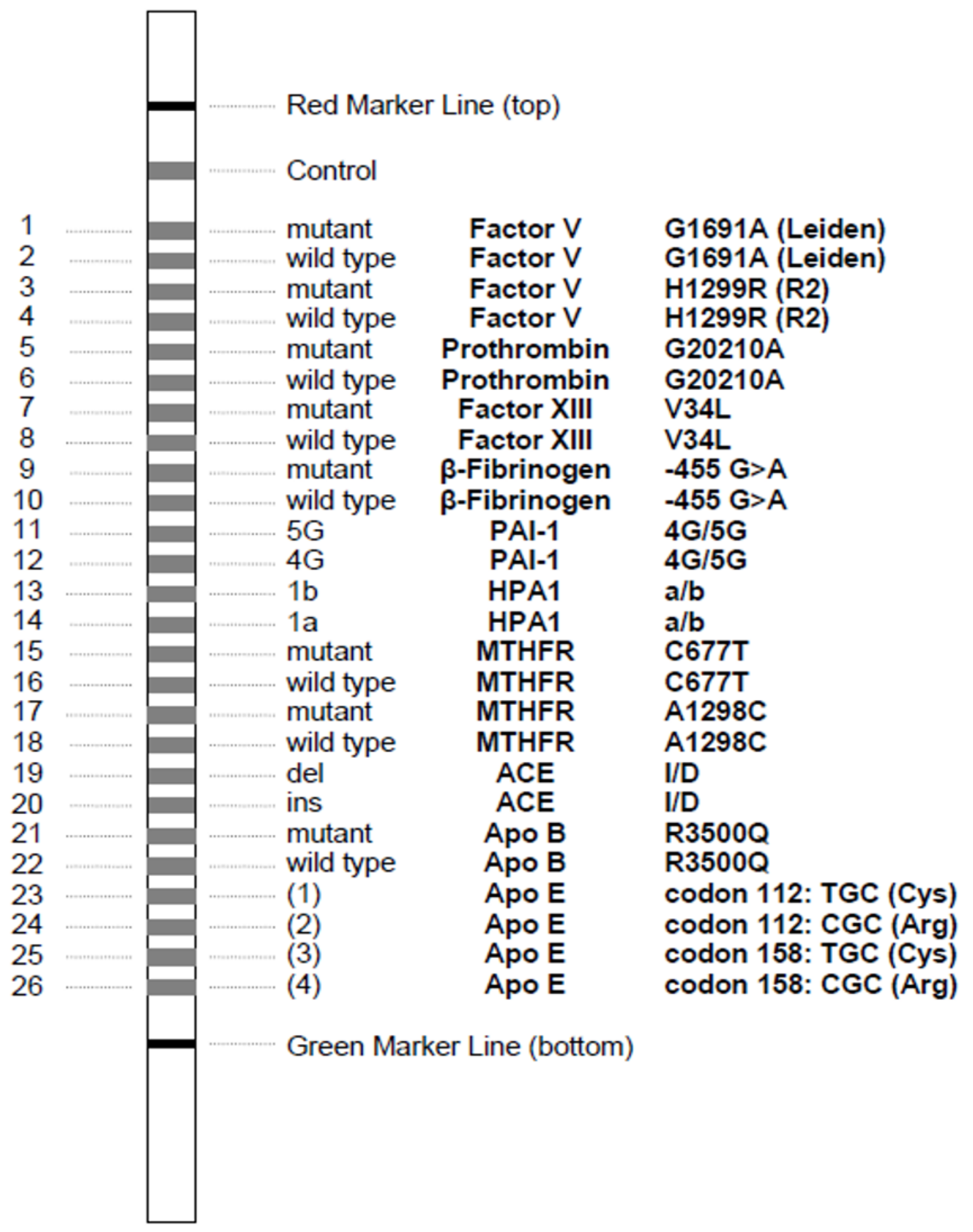

Figure 1

Test strip for detection of allele - specific hybridization 


\section{GENOTYPES}

NOR HET HOM

mutant line

$\square \quad \square \quad \square$

Fig. 2a

\begin{tabular}{|c|c|c|c|}
\cline { 2 - 4 } \multicolumn{1}{c|}{} & wild type line & mutant line & genotype \\
\hline NOR & positive & negative & normal \\
\hline HET & positive & positive & heterozygous \\
\hline HOM & negative & positive & homozygous mutant \\
\hline
\end{tabular}

Figure 2

Possible results of a test strip

E2 (112: Cys, 158: Cys)

E3 (112: Cys, 158: Arg)

E4 (112: Arg, 158: Arg) lines $(1)+(3)$

lines $(1)+(4)$

lines $(2)+(4)$

\section{APO E GENOTYPES:}

$\begin{array}{llllll}E 2 / 2 & E 2 / 3 & E 2 / 4 & E 3 / 3 & E 3 / 4 & E 4 / 4\end{array}$

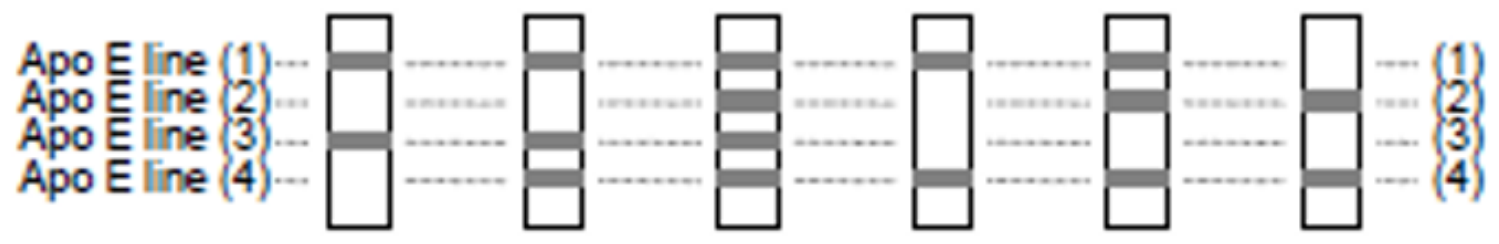

Figure 3

Possible results of a test strip for Apo E 


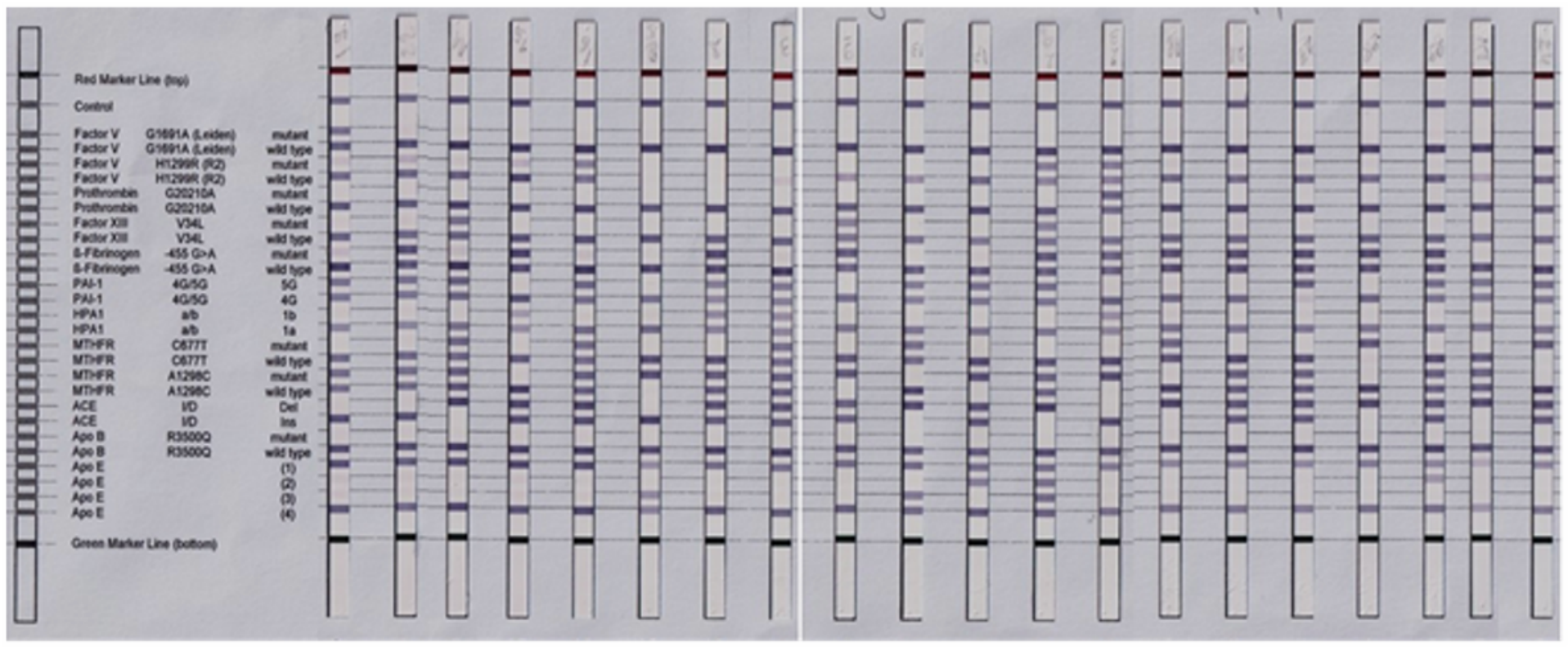

Figure 4

Results from the genotyping of 12 genetic variants in risk genes in diabetic patients with CVD

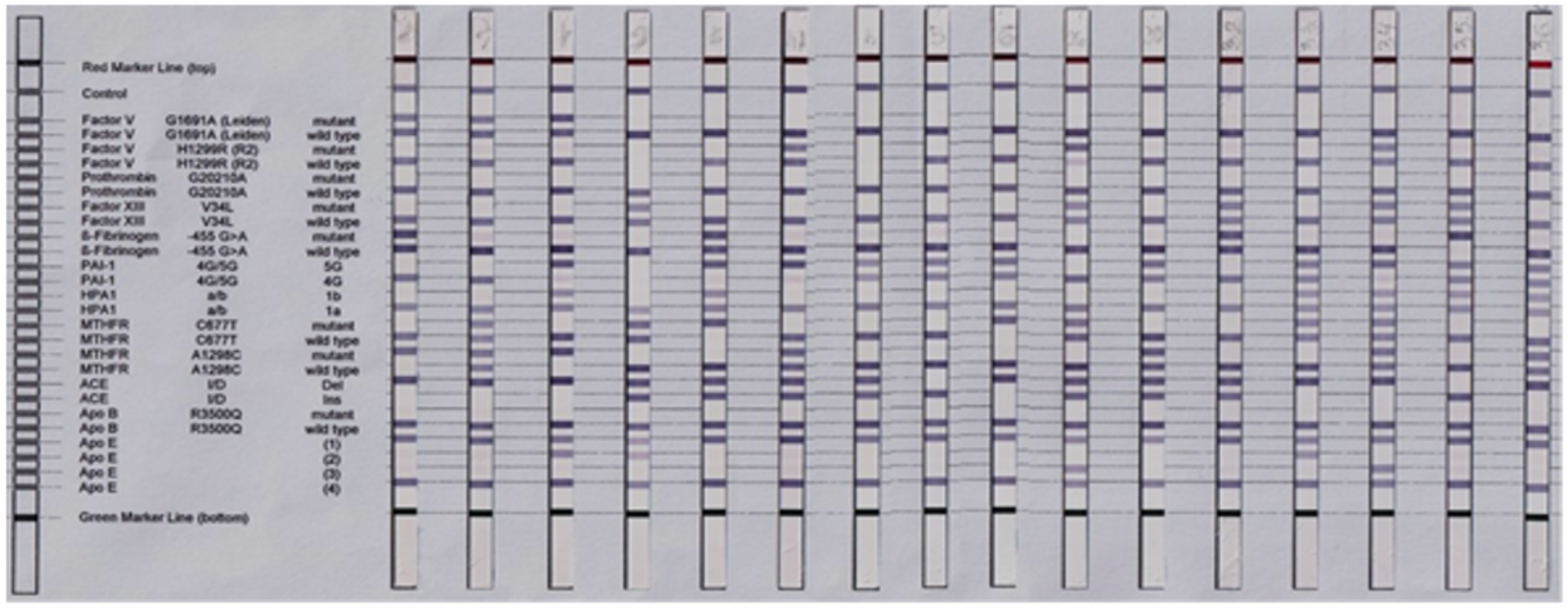

\section{Figure 5}

Results from the genotyping of 12 genetic variants in risk genes in diabetic patients without CVD
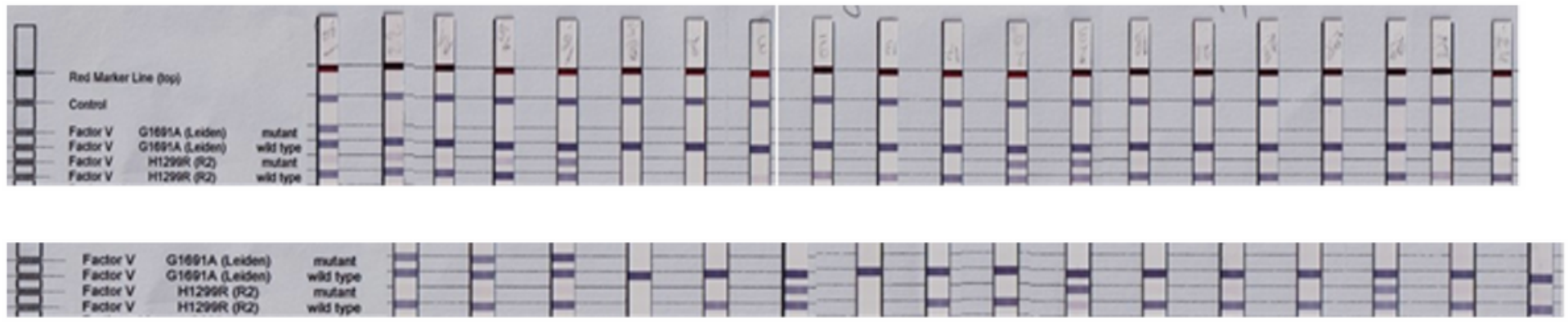


\section{Figure 6}

Results from the genotyping of FV G1691A (Leiden) and FV H1299R (R2) in patients with DM with CVD (top) and without CVD (bottom)
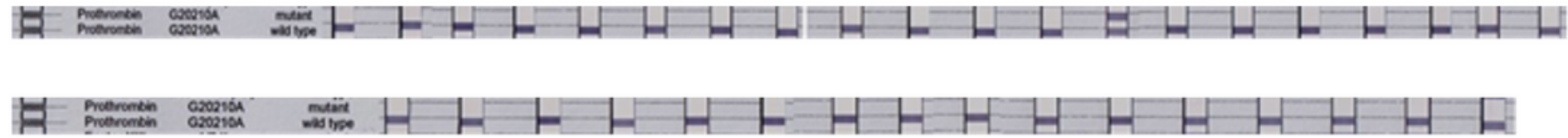

\section{Figure 7}

Results from the genotyping of Prothrombin G20210A in patients with DM with CVD (top) and without CVD (bottom)
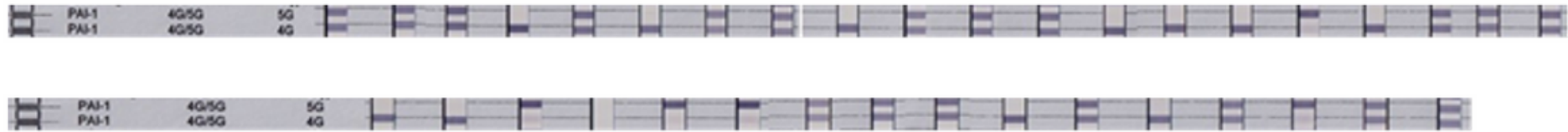

\section{Figure 8}

Results from the genotyping of PAl-1 4G/5G in patients with DM with CVD (top) and without CVD (bottom)
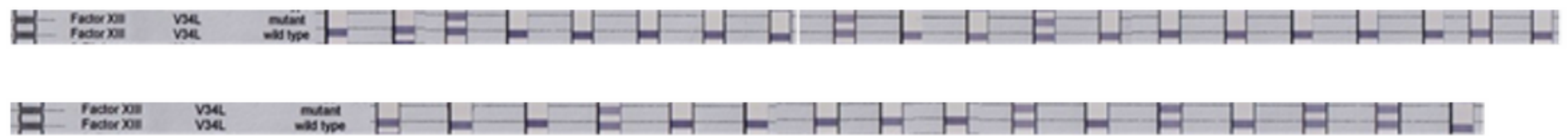

\section{Figure 9}

Results from the genotyping of Factor XIII V34L in patients with DM with CVD (top) and without CVD (bottom)

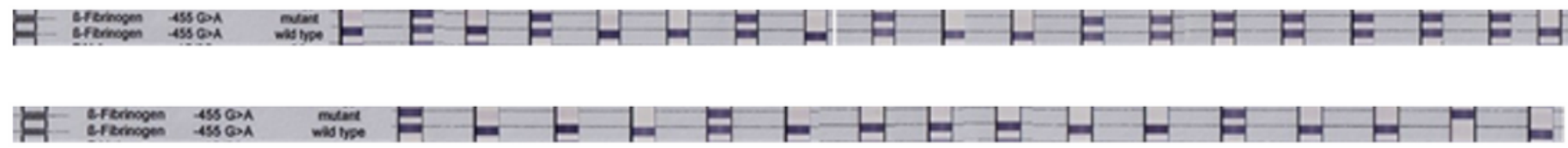

\section{Figure 10}

Results from the genotyping of $\beta$-Fibrinogen $-455 \mathrm{G} / \mathrm{A}$ in patients with DM with CVD (top) and without CVD (bottom) 

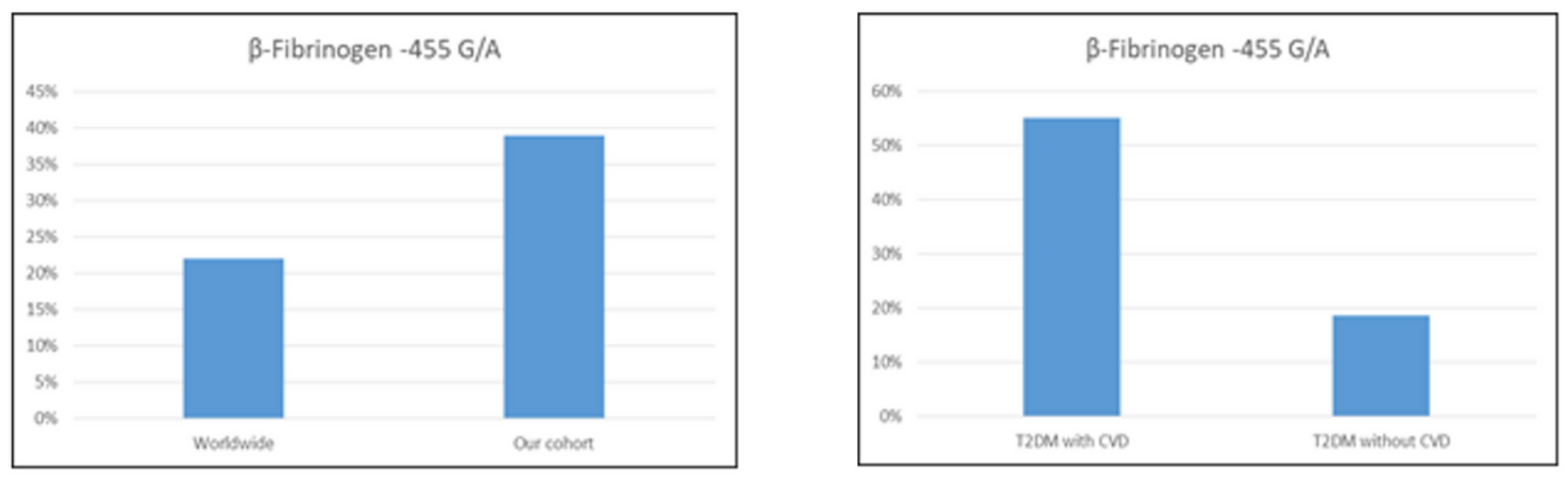

\section{Figure 11}

Frequency of the heterozygotes of the mutation for fibrinogen
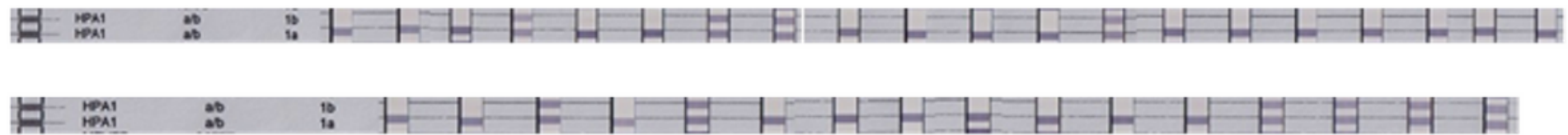

Figure 12

Results from the genotyping of GPIIla L33P (HPA-1) in patients with DM with CVD (top) and without CVD (bottom) 

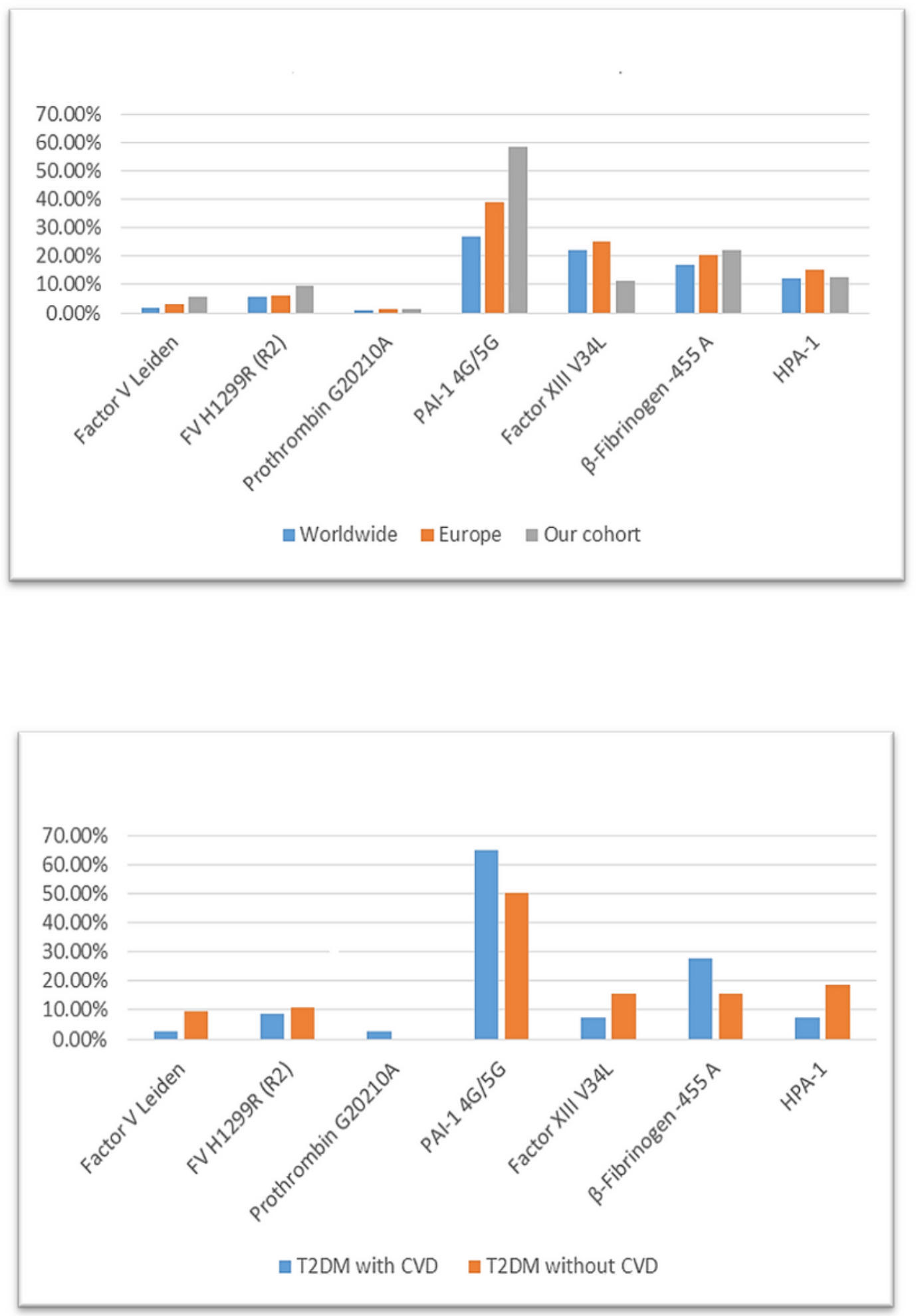

\section{Figure 13}

Frequencies of the studied variants of congenital thrombophilia in the different groups and populations. 

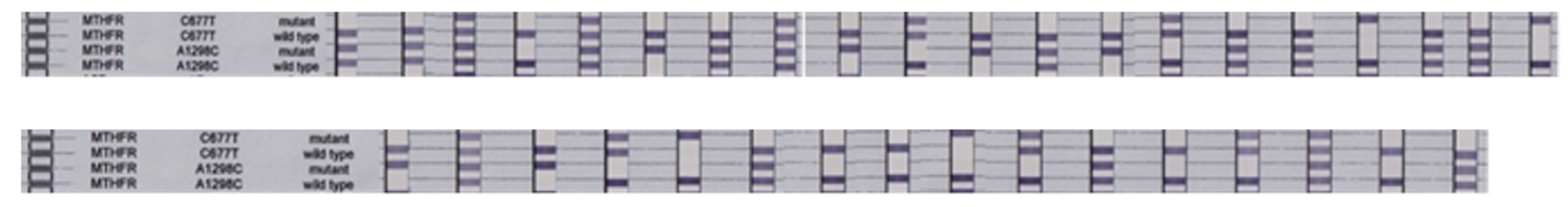

\section{Figure 14}

Results from the genotyping MTHFR C677T и MTHFR A1298C in patients with DM with CVD (top) and without CVD (bottom)
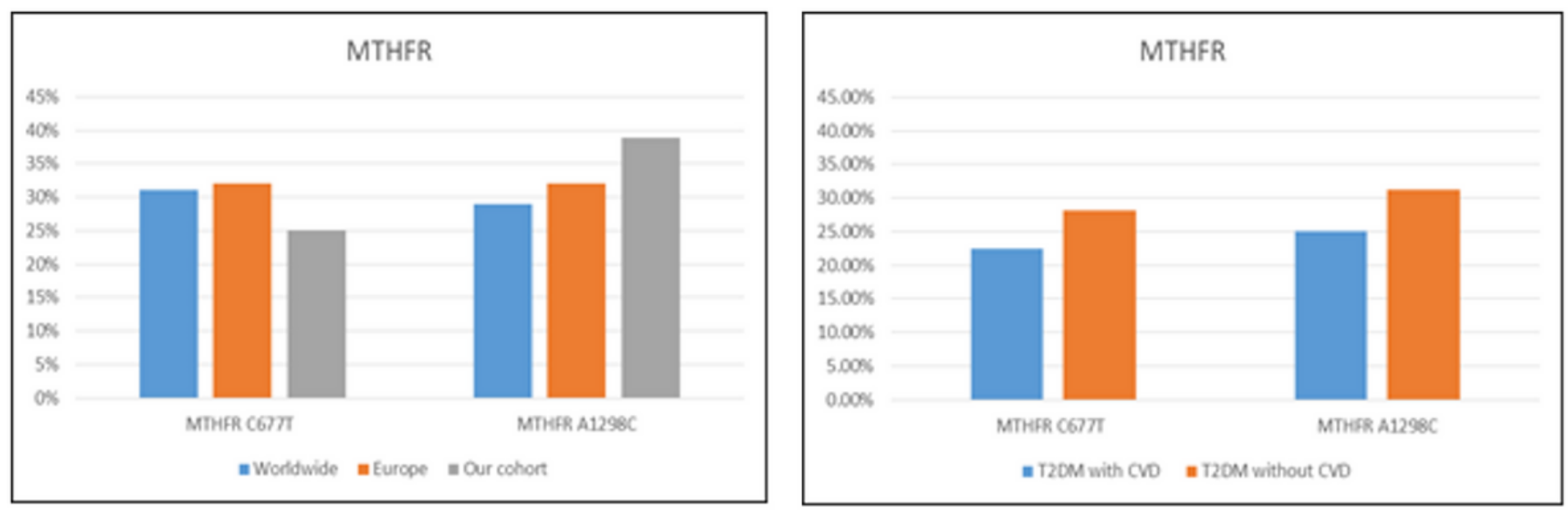

Figure 15

Allelic and genotypic frequencies of MTHFR C677T и MTHFR A1298C
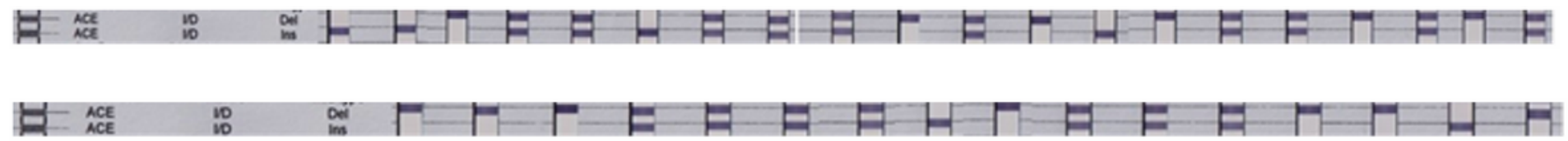

Figure 16

Results from the genotyping ACE I/D in patients with DM with CVD (top) and without CVD (bottom)
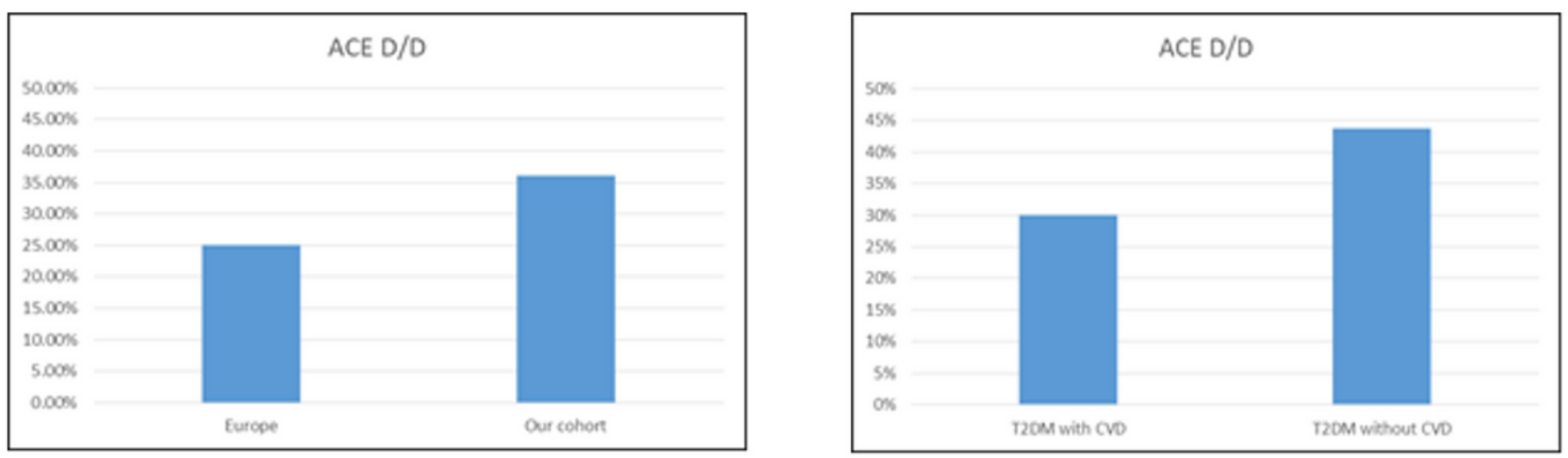
Figure 17

Allelic and genotypic frequencies of ACE I/D

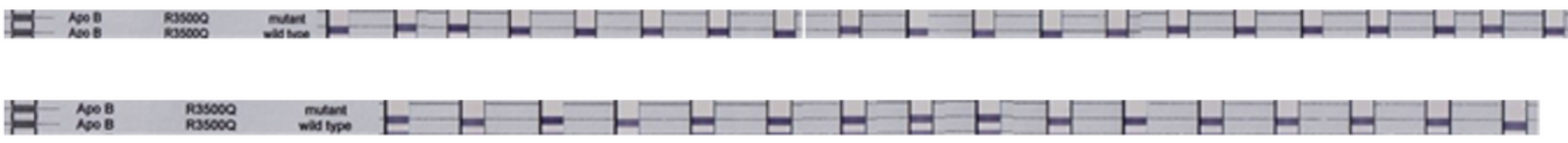

\section{Figure 18}

Results from the genotyping Apo B R3500Q in patients with DM with CVD (top) and without CVD (bottom)

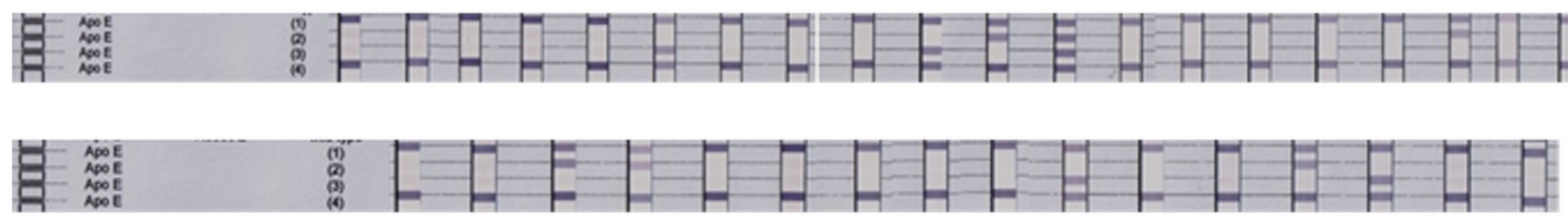

\section{Figure 19}

Results from the genotyping Apo E2/E3/E4 in patients with DM with CVD (top) and without CVD (bottom)
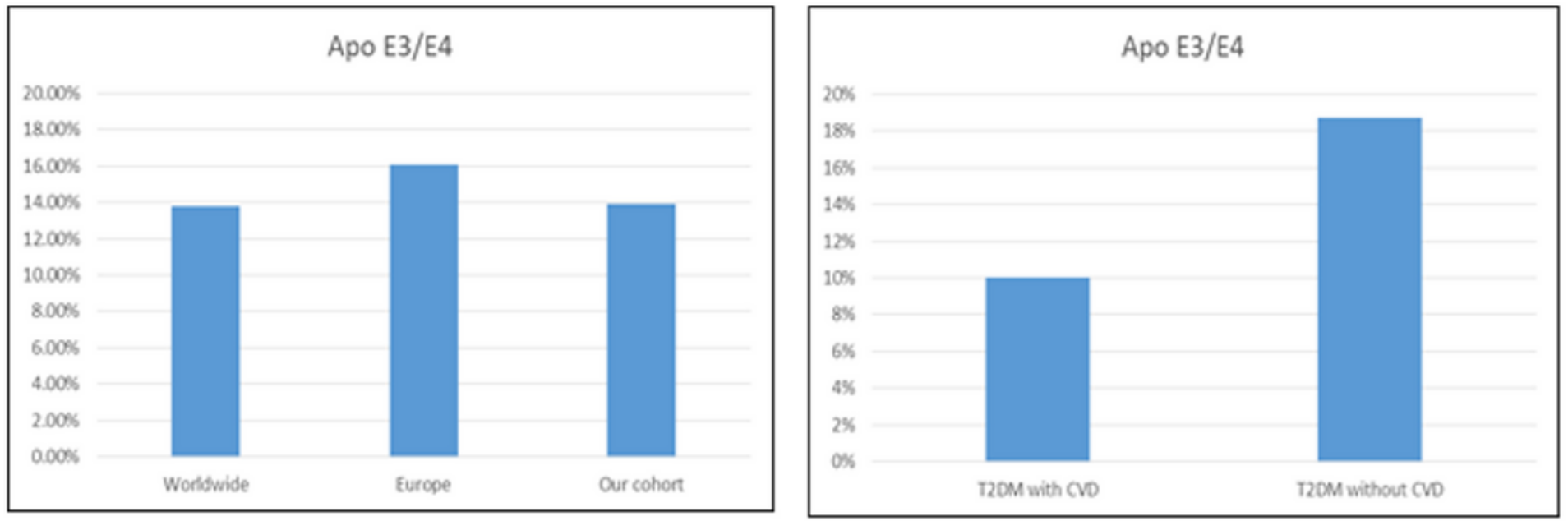

Figure 20

Allelic and genotypic frequencies of Apo E2/E3/E4 